\title{
La Biblioteca Pública en México: institución social para el fomento de la lectura y el libro
}

\author{
The Public Library in Mexico: Social Institution for Reading and Book Promotion \\ María Camila Restrepo Fernández \\ Posgrado en Bibliotecología y Estudios de la Información \\ Universidad Nacional Autónoma de México
}

Recibido: 14 de setiembre de 2019

Aceptado: 06 de marzo de 2020

Publicado: 18 de junio de 2020

\begin{abstract}
Resumen
El objetivo de este trabajo es demostrar el rol de la biblioteca pública como institución social, a partir del caso de la Ley de Fomento para Lectura y el Libro en México. Primero, se ahonda en la acepción de biblioteca pública como institución con funciones sociales, a través de la exploración de conceptos aportados por organismos internacionales, entes oficiales y académicos. Posteriormente, se revisan los hitos históricos en la conformación de la biblioteca pública en México para comprender el porqué del estado actual de las bibliotecas del país; se enuncian las legislaciones vigentes en materia de biblioteca pública y se focaliza la discusión en la Ley de Fomento para la Lectura y el Libro en relación con la biblioteca pública.
\end{abstract}

Palabras clave: Biblioteca pública, Fomento a la lectura, Ley de Fomento para la Lectura y el Libro, México.

\begin{abstract}
The main goal of this paper is to demonstrate the role of the public library as a social institution, based on the case of Reading and book promotion Law in Mexico. First, review the meaning of public library as an institution with social functions, through the exploration among the concepts contributed by international organizations, official and academic entities. Then, the historical landmarks in the conformation of the public library in Mexico are reviewed, in order to understand the current state of the libraries of the country, just as the applicable law about public libraries and finally focus the discussion is focused in Reading and book promotion law in Mexico.
\end{abstract}

Keywords: Public Library, Reading Promotion, Reading and Book Promotion Law, México. 


\section{Introducción}

A lo largo de la historia se ha situado la institución bibliotecaria como portadora y transmisora de la información registrada con el propósito de preservar y dar acceso a ella a las generaciones presentes y futuras; en especial se resalta una de sus tipologías: la biblioteca pública, la cual ha recibido por unanimidad social funciones que contribuyen al desarrollo e impulso de transformaciones sociales, culturales, educativas y políticas, porque presta servicios y ofrece bienes de información gratuitos, sin restricciones y en condiciones igualitarias para toda la población.

El estudio del estado de la Biblioteca Pública como institución, más allá de las adscripciones geográficas, adquiere relevancia en el contexto latinoamericano, porque es esta la institución de orden público que goza de mayor reconocimiento social y la revisión crítica a su origen, desarrollo y porvenir. Es, a la vez, una vía para detentar las características sociales de un pueblo. En los párrafos subsiguientes se ahonda sobre las tres etapas mencionadas en el contexto bibliotecario público mexicano, teniendo como eje principal la noción de Biblioteca Pública como institución social.

\section{La Biblioteca Pública como institución social}

Existe una multiplicidad de acepciones sobre la biblioteca pública de la que no pocos autores han propuesto aproximaciones a una definición total como institución; sin embargo, lo único permanente sobre la biblioteca pública es que siempre está evolucionando, pues debe responder e ir al unísono de las necesidades sociales que se generan espontánea o estructuralmente en el entorno social en el que se sitúa, así lo reafirma Shera (1949) al decir:

Cualquier intento por definer con precisión el término "biblioteca pública" genera confusion, ya que la institución es en sí misma una mezcla de intereses, objetivos y formas. El significado de 'biblioteca pública' cambia a medida que la institución depende del impacto de los cambios sociales y económicos, así como adquiere diferentes implicaciones y connotaciones con el paso del tiempo (1949, p. 157).

Por lo tanto, es fútil querer admitir una única y permanente definición para la biblioteca pública; lo que sí es estable son sus funciones y características porque sin importar el 
contexto, son estos dos elementos los que le dan identidad. Las principales características de la biblioteca pública son dos: la primera es la accesibilidad, este rasgo es esencial a la naturaleza de la institución de proveer "un acceso libre y sin límites al conocimiento, el pensamiento, la cultura y la información" (IFLA/UNESCO, 1994). La accesibilidad puede subdividirse en cinco tipos, a saber: social, todos sin distinción de sexo, raza, edad, género, idioma, religión, condiciones físicas o educación pueden acceder a la biblioteca y sus servicios; moral, no hay cabida a la censura de ninguna índole; física, la biblioteca debe ofrecer condiciones de infraestructura para todo tipo de personas a fin de que no pueda representar una barrera en el acceso; técnica, debe poseer herramientas y formación suficiente para que los usuarios hagan uso óptimo de los servicios y programas y debe tener accesibilidad intelectual; es decir, debe estar preparada para recibir y formar a la población usuaria cualquiera que sea su nivel educativo/cognitivo.

La segunda característica, quizás la más sustancial, es que la biblioteca pública es una institución financiada total o parcialmente por el Estado o alguna autoridad territorial pública, pueden existir bibliotecas con financiación híbrida, pero para ser considerada pública debe tener alguna participación estatal en su financiamiento y regulación.

Son los dos rasgos anteriores los que distinguen a la biblioteca pública entre las otras tipologías bibliotecarias; por ejemplo, para la biblioteca universitaria, la misión es servir de apoyo al currículo y a la comunidad universitaria, por lo que a diferencia de la biblioteca pública puede negarse a dar servicio a personas externas a su comunidad e incluso descartar solicitudes de información arguyendo que no está dentro del currículo académico.

En lo que respecta a las funciones de la biblioteca pública cabe aclarar que solo nos remitiremos a aquellas de orden social, pues partimos de la premisa que se trata de una institución social. La principal función social de la biblioteca pública es servir como fuente y medio de acceso a la información registrada, abriendo posibilidades de transformación individual y colectiva mediante la formación que da la biblioteca pública a sus usuarios con fines de creación y consolidación de hábitos de lectura y, más actualmente, de instrucción en habilidades informativas para la manipulación de las Tecnologías de la Información y la Comunicación (TIC); este proceso condensa la gran función social de la biblioteca pública: 
la transformación social a través de la lectura y el acceso libre a la información, la cultura y la educación.

Continuando con las funciones de la biblioteca pública, desde la mirada profesional e internacional, UNESCO/IFLA (1994);le otorga doce misiones a la biblioteca pública, siendo la primera una reiteración de lo mencionado en el párrafo inmediatamente anterior "Crear y consolidar los hábitos de lectura en los niños desde los primeros años" (IFLA, 2001, p. 72); es decir, en el panorama general, la biblioteca pública ostenta una posición de altísima responsabilidad, casi al nivel de instituciones como el Estado, la escuela o la familia. Por ejemplo, en la quinta misión, se dice que la biblioteca pública debe: "sensibilizar respecto del patrimonio cultural y el aprecio de las artes y las innovaciones y logros científicos" (IFLA, 2001 1994, p. 72). Del enunciado anterior queda implícito que la biblioteca pública es portadora de una parte significativa del patrimonio cultural, las innovaciones científicas y posee o debe tener las herramientas para fomentar el acceso al conocimiento.

El breve señalamiento anterior revela cómo la biblioteca pública ha sido atiborrada de funciones paradigmáticas que no puede cumplir a cabalidad, o bien podría hacerlo trabajando mancomunadamente con otras instituciones como museos, escuelas, universidades, ONGs, entre otros, y aun así sería difícil comprobar si tiene o no éxito en tal misión.

Ya en el plano estatal, las funciones sociales otorgadas a la biblioteca pública siguen siendo excesivas en relación con el apoyo económico y político que perciben por parte del Estado, especialmente en el precario sustento legislativo que se les da a las bibliotecas.

En el caso de América Latina las legislaciones sobre biblioteca pública son estrictas al definir qué es la biblioteca pública, exigiendo un número mínimo de ejemplares y servicios de alta calidad, al mismo tiempo que omiten dictar ordenanzas para asegurar y aumentar los recursos financieros destinados a la biblioteca pública. Por ejemplo, en algunas legislaciones nacionales como es el caso de Colombia, se exige un horario mínimo de operación a las bibliotecas públicas de 40 horas (Flores et. al., 2011, p. 11), mientras que en otras regulaciones como la mexicana, no se dicta ningún mínimo de horas de funcionamiento. En suma, exigencias como la mencionada, no se encuentran equiparadas en la misma 
legislación con alguna medida que la garantice, como bien podría ser el aseguramiento de la formación continua del personal o incluso la obligatoriedad de designar a profesionales del área bibliotecológica para dirigir las bibliotecas.

En el caso de la legislación vigente en materia de libros, lectura y bibliotecas en México, se puede comprobar que aunque se dictan disposiciones no hay claridad sobre qué agencia se hará cargo de llevarlas a cabo, aunque se enuncian las autoridades competentes para hacerlo, no es concreta la ley al indicar cuál entidad será titular de la responsabilidad.

En el caso de la Ley de Fomento para la Lectura y el Libro se listan cinco entidades gubernamentales distintas como responsables de dar cumplimiento al objeto de la ley, a saber, la Secretaría Cultural, la Secretaría de Educación Pública, el Consejo de Fomento para el Libro y la Lectura y, finalmente, los Gobiernos de las entidades federativas, municipales y demarcaciones territoriales de la Ciudad de México. Ciertamente, esta multiplicidad de actores para una única función crea desconcierto sobre quién realizará las acciones necesarias para dar fin cumplimiento a la ley.

Desde el redil teórico latinoamericano sobre biblioteca pública, autores como Civallero (2011), Jaramillo (2013) y Duque (2018) coinciden en que esta tiene como principal función reducir la exclusión social y favorecer la participación ciudadana, esta afirmación tiene sentido, porque en el contexto geográfico de análisis de los autores, existen casos particulares en los que se comprobó que la presencia de la biblioteca pública en una comunidad tuvo incidencia directa en la inclusión social de grupos minoritarios en dinámicas comunitarias como juntas de acción local, clubes de lectura y espacios culturales. El caso particular que más llama la atención se dio en Medellín, Antioquia (Colombia) y el estudio de caso fue realizado por la Dra. Orlanda Jaramillo, quien comprobó que la permanencia y operación de servicios y programas por parte del Parque Biblioteca España en el barrio Santa Cruz de la ciudad de Medellín, favoreció la inmersión en dinámicas comunitarias de grupos tanto excluidos como desplazados. Por lo tanto, existe un desbalance significativo entre lo que esperan las instituciones y los estados de las bibliotecas públicas y los medios que estas entidades le ofrecen a la biblioteca pública para dar cumplimiento a las expectativas, pese a que existen casos documentados en los que la biblioteca pública cumple un rol social muy destacado (Jaramillo, 2013). 


\section{Breve revisión: historia de la biblioteca pública en México}

La cultura escrita llegó a México por vías de la Conquista española al territorio mexicano en el Siglo XVI, materializada en libros y difundida, especialmente, por las órdenes religiosas que se emplazaron en el espacio conquistado. Gran parte de las bibliotecas que se establecieron en el primer siglo de la Colonia en Nueva España, permanecieron cerradas al público y servían casi exclusivamente a los integrantes de la comunidad religiosa, sin embargo, se cuenta como la primera biblioteca pública a la Biblioteca Palafoxiana, fundada en 1646 por el Obispo Juan de Palafox y Mendoza. El acervo de origen perteneció a su fundador y el propósito era formar al clero y dar acceso a la colección a cualquiera que quisiera conocerla, en esencia esta era una biblioteca pública porque no restringía el ingreso bajo ningún criterio, pero considerando el alto nivel de analfabetismo y la lengua de los textos, la prohibición estaba implícita, la comunidad de alfabetizados era mínima y muchos de ellos no dominaban la lengua castellana o el latín, por lo tanto, no tenían los medios para ingresar a la biblioteca y aunque se declarará pública no lo era a cabalidad (Zamora, 1994).

Desde inicios del siglo XIX y a lo largo del XX, el mundo a través de las instituciones y organizaciones civiles ha contribuido a alcanzar niveles de alfabetización óptimos. Es vital resaltar que, sin esta habilidad de decodificar el texto escrito, no serían factibles las funciones sociales de la biblioteca pública, especialmente cuando la pretensión principal es fomentar condiciones igualitarias en el acceso a los libros y la lectura.

Un segundo antecedente de la biblioteca pública en México es la Biblioteca de Catedral de México, también denominada como Turriana en honor a su fundador, esta se inauguró en 1788, por petición del arcedeán de la Catedral de México, el doctor Luis de Torres Tuñón, quien dispuso en su testamento que sus libros y veinte mil pesos fueran entregados al cabildo de México para la creación y manutención de una biblioteca con la condición explícita de que esta fuera pública (Bravo, 2008).

La Biblioteca del H. Congreso de la Unión es el tercer antecedente, cronológicamente ordenado, más relevante para la biblioteca pública en México, de naturaleza parlamentaria se instituyó en 1810 y desde entonces prestó servicio público. La Biblioteca del Congreso convivió con la Biblioteca de la Real y Pontificia Universidad hasta su clausura, una vez 
cerrada la Universidad, por decisión del Emperador Maximiliano, la Biblioteca del Congreso dio servicio a todos los estudiantes de la capital y los estados de la república en lugar de la extinta biblioteca de la Real y Pontificia Universidad. En 1936 el Congreso de la Unión formaliza la Biblioteca del H. Congreso de la Unión como biblioteca pública, teniendo así dos bibliotecas: una pública y otra parlamentaria, situadas en edificios independientes.

En el Siglo XX la figura de José Vasconcelos fue crucial para la conformación actual de las bibliotecas públicas, este prohombre propuso la creación de una Secretaria de Educación Pública y en ella un Departamento de Bibliotecas. Las obras que emprendió Vasconcelos como primer Secretario de Educación Pública (1921-1924) y luego rector de la Universidad Nacional Autónoma de México (1920-1921) continúan teniendo repercusiones en la forma en que se guían los procesos para la alfabetización y el fomento de la lectura en el país, siguen siendo concebidas, por muchos, como prácticas educativas y sociales que enmarcadas en la escuela y la biblioteca contribuyen a la transformación social y dan sustrato a la idea de que la educación debe ser la principal empresa del Estado (Ocampo, 2005), tal era la importancia otorgada por Vasconcelos (1998) a las bibliotecas que en El Desastre escribe:

La creación de un Departamento especial de Bibliotecas era una necesidad permanente, porque el país vive sin servicio de lectura y sólo el Estado puede crearlos y mantenerlos como un complemento de la escuela: la escuela del adulto y también del joven que no puede inscribirse en la secundaria y la profesional [...] También desde la escuela primaria operan juntos los tres departamentos encargados cada uno de su función: las ciencias enseñadas por la escuela propiamente dicha, la gimnasia, el canto y el dibujo a cargo de especialistas y no del mismo maestro normal, y la Biblioteca al servicio de todos, en sus diversos departamentos: infantil, técnico, literario, etc. (p. 6061)

Así queda por entendido que para Vasconcelos el papel de la biblioteca era de apoyo a la escuela, particularmente para la población adulta que no tenía acceso a la educación profesional y para aquellos que se encontraban en el nivel más elemental de instrucción; cabe destacar que fue el Mecenas de la Cultura el primero en concebir las labores bibliotecarias por departamentos o áreas para segmentar, no solo el trabajo del bibliotecario, sino como una visión diferenciada de los diferentes campos de acción de la biblioteca pública 
en la vida de la sociedad, incluyendo una fase administrativa hasta una antropológica, creando servicios diferenciados para niños, adultos, obreros y población indígena.

La concepción de interdependencia entre lectura y biblioteca que promovió Vasconcelos se ve expresada en los siguientes numerales de la Ley de Fomento para la Lectura y el Libro (2008): Promover el acceso y distribución de libros, fortaleciendo el vínculo entre escuelas y bibliotecas públicas (Art. 10, VII).

En esta ley expedida en 2008, con modificaciones del año en curso, continua y fortalece la relación antes mencionada por Vasconcelos en el Desastre, por lo que sugerir que la vigencia de sus ideas se mantiene hasta los días presentes no es exagerada, la estela de su trabajo se refleja en las iniciativas actuales. En esta misma Ley también se acepta la necesidad de dirigir los esfuerzos segmentando la población de acuerdo con sus intereses con la intención de asegurar un acceso igualitario, abierto y público a los programas para el fomento de la lectura, por ende, de acceso al libro a toda la población: garantizar la existencia de materiales escritos que respondan a los distintos intereses de los usuarios de la red nacional de bibliotecas públicas y los programas dirigidos a fomentar la lectura en la población abierta (Art. 11, IV).

Una de las estrategias promovidas por Vasconcelos y su grupo con miras a la alfabetización, la promoción de la cultura escrita y el fomento de la lectura fue El Libro y El Pueblo, esta publicación acercó -incluso después de su retiro como Secretario de Educación Pública- a población mexicana a la obra de valiosos escritores del país y difundió material para la formación de maestros y bibliotecarios a través de otra renombrada publicación, El Maestro Rural.

El Ministro Vasconcelos se rodeó de próceres de la historia de la educación en México como Ezequiel Chávez, Roberto Medellín, Francisco Figueroa, Francisco Morales y Jaime Torres Bodet, este último es quizás uno de los más prominentes y con mayor impacto en el campo de la lectura, los libros y las bibliotecas en México y el mundo, porque fue el impulsor del primer Manifiesto UNESCO sobre la biblioteca pública; desde entonces se declaraba la prioridad de asegurar la accesibilidad de todas las personas a la biblioteca pública sin ninguna limitante, la disponibilidad de recursos que satisfagan las necesidades e intereses 
de la población y se declaró en el Manifiesto UNESCO como esencia de la biblioteca pública el "ser fuerza viva de educación, cultura e información" (UNESCO/IFLA, 1994, p. 1).

Los pasos dados por Vasconcelos y su equipo en materia de bibliotecas públicas, bibliotecas escolares, alfabetización y fomento a la lectura fueron la punta de lanza para que en cada gobierno se le concediera a la biblioteca pública un espacio inamovible en la agenda gubernamental, así lo ejemplifica el rol llevado a cabo por María Teresa Chávez, impulsora de la literatura infantil, el fomento de la lectura con niños y pionera en la formación de bibliotecarios. Más avanzado el siglo XX emergen figuras notables como Ana María Magolini de Bustamante quien dirigió el establecimiento y operación de la Red Nacional de Bibliotecas Públicas de México, dejando cubierto el $88 \%$ de los municipios del país al final de su gestión, durante la que además aumentó el acervo total (catalogado y clasificado) de las bibliotecas públicas de 1,332,000 a 30,142,633 (Morales, 2001).

El rol de los bibliotecarios en la historia de la biblioteca pública en México ha sido fundamental, si bien es cierto que su presencia ha sido más fuerte en unas épocas que en otras, el quehacer bibliotecario ha logrado establecer unos mínimos para el correcto funcionamiento de las bibliotecas públicas en la nación. Desde la formación de bibliotecarios en el extranjero, como fue el caso de Juana Manrique de Lara, responsable de la introducción de las bibliotecas infantiles y juveniles en México, hasta el trabajo con comunidades indígenas en favor del rescate de su cultura; han sido los bibliotecarios un eje fundamental para la evolución y permanencia de la biblioteca pública en el país.

La historia de la biblioteca pública en México es extensa, igualmente lo es la historia de la lectura, porque desde unas centurias la necesidad de construir sociedades democráticas se convirtió en un propósito compartido, esto implica que los potenciales individuos que pasarán a formar parte de la anhelada sociedad democrática deberán reunir un conjunto de condiciones intelectuales mínimas que aseguren su justa participación, entre ellas y probablemente la más sobresaliente, es la lectura como práctica social enraizada a la biblioteca. 


\section{Marco actual legislativo de las bibliotecas públicas en México}

La normativa mexicana para las bibliotecas públicas es cuantiosa porque las hay de aplicabilidad nacional como la Ley de Fomento para la Lectura y el Libro o la Ley General de Bibliotecas, como también existen regulaciones restringidas a las ciudades, tal es el caso de la Ley de Bibliotecas del Distrito Federal o los compendios correspondientes a los estados, por ejemplo: Ley de bibliotecas del Estado de Jalisco, Ley del libro y las bibliotecas del Estado de Durango, entre otras, contando además, con regulaciones menores como decretos y reglamentos que se viabilizan a las normas superiores.

En términos generales, la legislación sobre bibliotecas es estable, teniendo en cuenta la longevidad de la Ley General de Bibliotecas, emitida en 1988, y la Ley de Fomento para la Lectura y el Libro que ya cumple once años de vigencia y será abordada en el apartado siguiente.

\section{a. Ley de Fomento para la Lectura y el Libro}

La Ley de Fomento para la Lectura y el Libro fue promulgada el 23 de julio de 2008 por el presidente Felipe Calderón, esta norma surgió como parte de una discusión que se extendió durante varias legislaturas del Congreso sobre regulaciones relativas al libro, por ende, a la lectura. El antecedente inmediato de la Ley de Fomento es la Ley del libro aprobada en 2000, aunque nunca entró en vigor puesto que no se conformó el Consejo Técnico que expediría el reglamento (Rodríguez, 2008).

Sin embargo, la historia de la Ley de Fomento va más atrás de la mencionada Ley de Libro aprobada en 2000; en la LVI legislatura del Congreso de la Unión, la diputada Margarita Villanueva Ramírez presentó ante la Cámara de Diputados una iniciativa de ley que sirviera para fomentar el consumo de libros entre la población, apoyándose en el descenso en ventas de libros, pese al vertiginoso aumento poblacional, es decir, aunque la población aumentaba, la producción y compra de libros disminuía, el argumento que presentó Villanueva Ramírez era contundente, sin embargo, no llegó a materializarse durante la LVI legislatura, pero sentó un precedente para las legislaturas siguientes. 
La Ley de Fomento contiene los siguientes puntos neurálgicos en relación con las funciones sociales de la biblioteca pública: la obligación de la Secretaria de Educación Pública en la dotación de acervos para las bibliotecas de aula y escolares, como forma para asegurar la subsistencia de esa tipología bibliotecaria; ordena el establecimiento del Consejo Nacional de Fomento para el Libro y la Lectura, estamento encargado de crear y vigilar el reglamento de funcionamiento; dictamina la creación de espacios para la concertación entre los diferentes actores de la cadena de producción y divulgación del libro, a saber, autores, editores, impresores, papeleros, distribuidores, libreros y bibliotecarios, inclusive los lectores, y finalmente, establece el precio único o regulación comercial como forma de asegurar un acceso igualitario al libro.

El primer punto, la obligación de la Secretaria de Educación Pública en la dotación de acervos para las bibliotecas de aula y escolares, el estado actual de la biblioteca escolar en México es incierto, de acuerdo con el Informe de la OEI y la Secretaria de Educación Pública de México (2010), el desarrollo ha sido desigual entre bibliotecas escolares y son pocas las que gozan de reconocimiento por parte de la comunidad educativa en la que están inmersas.

La tenencia o no de prestigio está directamente relacionada con el nivel formativo de los bibliotecarios, la calidad de los acervos, el espacio, el equipamiento y los servicios de información que ofrecen a los integrantes de las instituciones educativas. Especialmente la carencia de acervos de calidad y suficientes para apoyar e integrarse debidamente al currículo escolar es lo que ocasiona el aislamiento de la biblioteca escolar de las dinámicas escolares, aquellas que cuentan con colecciones ricas en relación con sus títulos, no están debidamente organizadas o su estado físico no es óptimo.

Debido a las condiciones mencionadas, la necesidad de una sinergia entre la biblioteca escolar y la biblioteca pública es imperativa para dar cumplimiento a la Ley de Fomento, porque la biblioteca pública puede aportar parte de sus colecciones y servicios para satisfacer necesidades e intereses informativos, exceptuando los libros de texto, que por antonomasia no deben hacer parte del acervo de una biblioteca pública. Torres Bodet sintetiza con absoluta precisión la necesaria relación entre ambas bibliotecas así: "la escuela y la biblioteca no deben considerarse como instituciones rivales; ni siquiera como entidades 
independientes. Si una y otra no se articulan, nuestro proceso será muy lento" (OEI y Secretaria de Educación Pública, 2010, p. 7).

En el segundo punto, relativo al Consejo Nacional de Fomento para el Libro y la Lectura, se define como un "órgano consultivo de la Secretaría de Cultura y espacio de concertación y asesoría entre todas las instancias públicas, sociales y privadas vinculadas al libro y la lectura" (México. Cámara de Diputados, 2008, p. 5). Este órgano está compuesto por quince integrantes de nivel directivo provenientes de los siguientes estamentos gubernamentales: Secretaria de Cultural, Secretaria de Educación Pública, titular del Instituto Nacional de Lenguas Indígenas, Presidente de la Cámara Nacional de la Industria Editorial Mexicana, Director General de Materiales Educativos de la Secretaría de Educación Pública, Director General de Bibliotecas de la Secretaría de Cultura, Director General del Instituto Nacional del Derecho de Autor, Director General de la Comisión Nacional de Libros de Texto Gratuitos, Presidente de la Comisión de Biblioteca y Asuntos Editoriales de la Cámara de Senadores y Presidente de la Comisión Bicameral del Sistemas de Bibliotecas del Congreso de la Unión; también tiene una representación la entidad mixta Fondo de Cultura Económica con la inclusión del Director General en el Consejo y, finalmente, por parte de los estamentos civiles o académicas tienen asiento los siguientes representantes: el Presidente de la Asociación de Libreros de México, el Presidente de la Asociación Nacional de Bibliotecarios y el Presidente de la Sociedad General de Escritores de México. Además, podrán participar de las sesiones del Consejo, personas y otras entidades no mencionadas, siempre que el mismo Consejo considere que la participación de entidades o personas externas contribuirá al cumplimiento de las funciones.

La función sustantiva del Consejo es crear y ejecutar el Programa de Fomento para la Lectura y el Libro, cuya meta es promover sistemas integrales para el acceso a la lectura y el libro convenidos equilibradamente entre el sector público, privado y civil. En relación directa con la biblioteca pública, este Consejo en su Programa dicta los lineamientos fiscales, jurídicos y administrativos para el fomento de la lectura; esta función se duplica con las directrices dadas por otros estamentos nacionales y de los estados, en especial con la Secretaria de Cultura, que aunque presida el Consejo, tiene otros departamentos administrativos con funciones dictaminadoras sobre las bibliotecas públicas, aunado al 
hecho de que en los estados se duplica la legislación, porque la mayoría de los estados tienen leyes y decretos propios para controlar la función de las bibliotecas públicas.

El Programa de Fomento en vigor (2016-2018) refiere como objetivos para la biblioteca pública el fortalecimiento de los acervos de la Red Nacional de Bibliotecas Públicas, sobre todo aquellas que dan servicio al estudiantado de nivel básico, es decir, desde el Consejo existe la conciencia sobre la función de la biblioteca pública como complemento a la biblioteca escolar y en los casos más extremos como la única alternativa para los escolares. La formación continua de los bibliotecarios públicos también es una de las finalidades del Programa, las capacitaciones se hacen mediante talleres, seminarios, cursos y encuentros planeados y ejecutados mancomunadamente entre las diferentes entidades que hacen parte del Consejo, sin embargo, el Programa de Fomento no precisa un plan concreto y cronológico de las actividades formativas, esta situación podría ser causa del alto número de entidades implicadas y la plausible multiplicidad de responsabilidad que tal situación puede ocasionar.

En lo referente a la creación de espacios para la concertación entre los diferentes actores de la cadena de producción y divulgación del libro, la responsabilidad del Consejo es mediar entre los actores que tienen representación en el órgano, esta labor no considera explícitamente la participación de la sociedad civil quienes serán los receptores finales de las actividades y espacios incentivados por el Consejo, además, en la misma Ley de Fomento, en el Capítulo IV, responsabiliza a la Secretaria de Cultura como la instancia responsable de coordinar los espacios, programas, proyectos y acuerdos entre entes públicos, privados y civiles, a la vez que crea en la legislación al Consejo y le otorga funciones similares. Así en Art. 20 No. III, es responsabilidad de la Secretaria de Cultura:

Establecer compromisos con las instancias y organismos internacionales que, mediante convenios y acuerdos bilaterales y multilaterales, incentiven el desarrollo integral de las políticas públicas en la materia facilitando a autores, editores, promotores, lectores, espacios y alternativas de promoción y difusión que favorezcan el conocimiento de nuestra obra editorial y literaria en el exterior. (México. Cámara de Diputados, 2008, p. 8) 
Y al Consejo le otorga la misma responsabilidad en el Art. 15 No. III

Concertar los esfuerzos e intereses de los sectores público y privado para el desarrollo sostenido de las políticas nacionales del libro y la lectura.... Proponer a las autoridades competentes la adopción de políticas o medidas jurídicas, fiscales y administrativas que contribuyan a fomentar y fortalecer el mercado del libro, la lectura y la actividad editorial en general. ((México. Cámara de Diputados, 2008, p. 7)

En síntesis, la Ley no es taxativa en cuanto a funciones y responsabilidades, error u omisión que podrían afectar tangencialmente el objeto de la Ley. Por último, el precio único, la Ley de Fomento establece una tarifa común de precio de venta en cualquier lugar del territorio mexicano, el precio es definido por el productor del libro y no por algún órgano de gobierno, este último solo reglamenta la estabilidad del costo al público de una publicación. El precio único es el punto que ha generado mayor respuesta por parte de los medios de comunicación, librerías, editoriales y organismos académicos respecto a la Ley de Fomento, con esta ordenanza el ente oficial busca crear condiciones equitativas en el acceso al libro, evitando la competencia desleal entre librerías y editoriales para que el mercado del libro se oriente más a ofrecer riqueza en los títulos y no en las ofertas comerciales, puesto que la Ley se encamina a mejorar tanto las condiciones de los lectores como las condiciones siempre cambiantes e inestables de la industria editorial.

En términos generales, la percepción sobre el impacto del precio único en el mercado librario es positiva, pero personajes de la industria editorial como Anaya Rosique consideran que:

[...] la ley tiene criterios para el fomento de librerías y eso es algo que nos hace falta. Hablamos del mercado del libro, de poner precio único al libro durante un periodo determinado para que haya una competencia sobre bibliodiversidad, sobre calidad y contenidos más que sobre descuentos, pero falta hablar de librerías" (Aguilar, 2017).

La mención de Rosique a las librerías se debe al impacto de la Ley en el mercado, que de no ser estrictamente vigilada termina por depredarse a sí misma, porque regula los precios, pero no tiene ninguna disposición explícita para incentivar el desarrollo de nuevas librerías. Para las bibliotecas públicas el precio único repercute en la adquisición de colecciones que se hacen desde la Red Nacional de Bibliotecas Públicas, a la fecha no ha habido 
pronunciamientos por parte de esta organización sobre el impacto del precio único en la adquisición de material bibliográfico.

México comparte con los países de la región la intención de aumentar sus lectores, partiendo de la idea de que el hábito lector actual es pobre en calidad y cantidad, por lo que un primer paso para tener una nación de lectores es crear, emitir y vigilar normas que fomenten la lectura y creen condiciones educativas, económicas, culturales y sociales para los organismos e instituciones que enarbolan la bandera de la lectura como la escuela, la biblioteca y la industria editorial, por supuesto, cada una con propósitos y medios diferentes, por ello una Ley con intenciones ecuménicas es de doble filo, a la larga podría favorecer a algún actor o entidad sobre otra y no cumplir el objeto inicial.

\section{A modo de cierre}

La Ley de Fomento para la Lectura y el Libro está dirigida con mayor fuerza al mercado editorial, de ahí que los puntos más controversiales son aquellos que afectan al mercado de producción y compra del libro, dejando a un lado el aspecto de fomento a la lectura, porque la premisa de que al tener mejores y más vías de acceso al libro va a aumentar el hábito lector es una imprecisión y no existen casos de orden nacional o regional que puedan dar prueba de ello.

En sentido estricto la Ley sí estimula la compra de libros, que según las estadísticas de la Cámara Nacional de la Industria Editorial Mexicana (CANIEM) entre 2011 y 2015 ha ido en aumento y las regresiones que ha tenido entre años no han sido altas, esto puede significar que sí ha habido un correcto estímulo en el mercado para aumentar la demanda libraria por parte del público y las instituciones (ver las estadísticas en http://www.caniem.com/content/actividad-editorial); sin embargo, no hay un método para contrastar las cifras de compra con el aumento cuantitativo y cualitativo de las prácticas lectoras de los ciudadanos mexicanos en relación con su uso de las colecciones y participación en los servicios que ofrecen las bibliotecas públicas del país.

Los objetos de la Ley consignados en su Artículo 4 son todos pertinentes, si se tratara exclusivamente de una norma dirigida a la industria editorial, pero su inclusión titular de 
fomento a la lectura sugiere, por tanto, que debería considerar y dictaminar artículos que contribuyan directamente al mejoramiento de las condiciones de operación y mantenimiento de las bibliotecas públicas mexicanas, porque son estas las que ofrecen el acceso democrático e igualitario a los ciudadanos que no tienen el poder adquisitivo, las condiciones sociales, educativas y culturales para acceder al libro y la lectura.

La intención gubernamental de fomentar la lectura se ve más reflejado en otras disposiciones de orden nacional como el Programa Nacional de Salas de Lectura y el Programa Nacional de Lectura, son estos dos programas -que deberían tener fuerza de ley para operar a plenitud- las iniciativas que más se orientan hacía la anhelada nación de lectores, solo a través del potenciamiento de instituciones sociales como la biblioteca pública, se puede propiciar un espacio justo y equitativo en el que tengan cabida integrantes de todos los colectivos sociales. De no fortalecer debidamente a la biblioteca pública se incurre en la ingenuidad que demostraron don Luis Torres de Tuñon o Juan de Palafox, al ofrecer ricos acervos bibliográfico a hombres y mujeres entre los que predominaba el analfabetismo, por ello para incentivar el mercado, primero se debe crear la demanda, esto es: fomentar la necesidad social de la lectura.

Los esfuerzos de la Ley deben dirigirse así a fortalecer el mercado editorial, pero debe hacerse reconociendo que la democratización del libro trasciende al aspecto comercial y requiere del desarrollo de estrategias educativas y culturales que se asemejen al servicio nacional de lectura que sugería Vasconcelos, como prioridad de la educación pública brindada a través de la escuela y la biblioteca, es probable que de esta manera se logre posicionar a la lectura y el libro como formas de ver, estar y participar del mundo.

\section{Referencias bibliográficas}

Aguilar, Y. (2017). Urge reformar la Ley de Fomento para la Lectura: expertos. El Universal. Recuperado de https://www.eluniversal.com.mx/cultura/letras/urge-reformar-la-leyde-fomento-para-la-lectura-expertos

Bravo, B. (2008). Los libros y los lectores en la biblioteca pública de la Catedral de México (1804-1867). Biblioteca Universitaria, 11(2). Recuperado de http://revistas.unam.mx/index.php/rbu/article/view/24641/23112 
Civallero, E. (2011). El rol de la biblioteca en la inclusión social. En XIII Jornadas de Gestión de la Información de SEDIC (Asociación Española de Documentación e Información), Madrid (España). Recuperado de http://www. aacademica.org/edgardo.civallero/51.pdf

Cámara de Diputados del Honorable Congreso de la Unión. (2008). Ley de Fomento para la lectura y el libro. México: Cámara de Diputados del Congreso de la Unión.

Duque, N., \& Mazón, V. (2018). Bibliotecas y desigualdad social: explorando el estado del arte. Anales De Documentación, 21(1). Recuperado de https://doi.org/10.6018/analesdoc.21.1.289021

Fernández de Zamora, R. (1994). La historia de las bibliotecas públicas en México: un tema olvidado. En 60th IFLA General Conference. Recuperado de https://originarchive.ifla.org/IV/ifla60/60-ferr.htm

Flores Faúndez, C., et. al. (2011). Legislación de Bibliotecas Públicas de America Latina. Análisis comparativo: Colombia-México-Uruguay-Venezuela. En XVI Conferencia Internacional de Bibliotecología, Santiago, Chile. Recuperado de http://eprints.rclis.org/17502/1/Legislacion\%20Bibliotecas\%20Publicas\%20A.L.\%20

\section{Cherie.pdf}

IFLA. (2001). Directrices IFLA/UNESCO para el desarrollo del servicio de bibliotecas $\begin{array}{llll}\text { públicas. } & \text { S.I.: } & \text { IFLA. }\end{array}$ https:/www.ifla.org/files/assets/hq/publications/archive/the-public-libraryservice/pg01-s.pdf

Jaramillo, O. (2013). Biblioteca pública, ciudadanía y educación social. Argentina: Alfagrama.

Morales, E. (2001). Nuestros bibliotecarios. Biblioteca Universitaria, 4 (2): 118-121. Recuperado de http://www.redalyc.org/pdf/285/28540208.pdf

Ocampo, J. (2005). José Vasconcelos y la educación mexicana. Revista Historia de la Educación Latinoamericana, 7, 139-159. Recuperado de http://www.redalyc.org/pdf/869/86900707.pdf

OEI y Secretaria de Educación Pública, México. (2010). Las bibliotecas escolares en México. Un diagnóstico desde la comunidad escolar. México: Secretaría de Educación Pública.

Recuperado de http://ideamex.com.mx/sites/default/files/pdf/bibliotecas\%20 escolares mexico.pdf 
Rodríguez, F. (2008). Una década de debate: la Ley para el fomento de la lectura y el libro en México en perspectiva comparada. Espacios Públicos, 11 (23). Recuperado de http://www.redalyc.org/articulo.oa?id=67611217012

Shera, J. (1949). Foundation of the public library. Chicago: University Chicago Press.

Vasconcelos, J. (1998). El desastre. México: Editorial Trillas.

\section{Notas de la autora}

María Camila Restrepo Fernández: estudiante del Posgrado en Bibliotecología y Estudios de la Información, Universidad Nacional Autónoma de México. Correo electrónico: camila0330.maria@gmail.com, ORCID: https://orcid.org/0000-0002-9043-3306 